\title{
Monitoring of Collaborative Assembly Operations: An OEE Based Approach
}

\author{
Sauli Kivikunnas, Esa-Matti Sarjanoja, Jukka Koskinen, and Tapio Heikkilä \\ VTT Technical Research Centre of Finland, Networked Intelligence, Kaitoväylä 1, \\ FI-90570 Oulu, Finland \\ \{Sauli.Kivikunnas, Esa-Matti.Sarjanoja, \\ Jukka.Koskinen, Tapio.Heikkila\}@vtt.fi
}

\begin{abstract}
In this paper we present requirements and concept generation principles for performance monitoring of a collaborative assembly task. Life cycle aspects are considered and an Overall Equipment Efficiency (OEE) based monitoring scenario for a developed passive collaborative robot (COBOT) test system is presented. In this case main benefits of applying COBOT are expected to be: improved productivity, improved quality, reduced production cost and improved ergonomics. Since human and COBOT are working cooperatively human actions have also affects on process performance, i.e. OEE. However a human's and machines or a COBOT's efficiency are undistinguishable directly from OEE factors. It is possible to infer cause of lower efficiency from the variables from which OEE factors are calculated. One such variable is cycle time, which is used to define performance efficiency.
\end{abstract}

Keywords: OEE, car rear screen installation, COBOT.

\section{Introduction}

Business environments are changing continuously leading to needs for changes in production and manufacturing. Production strategies need to be revised to be better able to satisfy changing customer needs. These demands can be met with technologies introducing different types of flexibilities into production.

Technologies for achieving these flexibilities imply changes in software, machinery and layouts. This paper contributes to increasing flexibility by introducing a performance monitoring concept for new machines or robots for assisting humans in assembly tasks.

Many current industrial manual assembly tasks could be fully automated with conventional robots, but high flexibility is often difficult to be achieved cost-effectively with conventional robot systems [1]. Robotic systems are usually cost-effective in assembling high volume products, but flexibility in these assembly lines is low. Low and medium volume products are typically customized for customer needs and production times and volumes may vary depending of a product demand. Therefore for low and medium volume products high product and mix flexibility are required by manufacturing systems. High flexibility can be achieved by co-operative robot-human systems, rather than by autonomous robots. 
In collaborative task operations the human operator takes care of controlling interactively all the critical operations with required accuracy targeting also to better quality. The effect of new device or any improvements on the assembly line performance should always be evaluated, also in the case of collaborative task execution. An increasingly common method for this is to use Overall Equipment Efficiency (OEE). It is an indicator how well equipment or machinery are performing or are being utilized at a time period and it indicates, which parts of assembly line or machinery are not performing well. OEE is a tool which helps in focusing improvement activities.

Life cycle costs are evaluated in planning and designing of manufacturing line or machines whereas OEE is typically used for benchmarking, evaluating and continuously improving performance of a manufacturing line or machines (process performance aspect). Co-operative assembly robots sharing a workplace with humans are called assistant robots. Such a robot can be guided physically by a human or it assists human worker without any physical guidance. In the previous case the robot can be called as a passive collaborative robot (COBOT) [2] and the latter case intelligent assists robots. A typical example of a COBOT application is an assembly task where human lifts a heavy load co-operatively with COBOT: human introduces motion intelligence and the COBOT produces power assistance. An assembly system that executes and monitors tasks where a robot and a human work co-operatively needs to be flexible. The systems should recognize and recover from abnormal situations, like safety risks or system malfunctions.

This paper presents requirements and a concept for performance monitoring of a collaborative assembly task. Life cycle aspects are considered and an OEE based monitoring scenario for a developed COBOT test system is presented.

\section{Theoretical Background}

According to [3] OEE can be used at three levels of manufacturing: manufacturing plant, manufacturing line and machine process level. In the manufacturing plant OEE can be used as benchmark before (initial performance) and after changes. At the manufacturing line level OEE can be used to indicate performance of the production line. In the machine process level OEE indicates performance levels of the machines.

Overall equipment efficiency is defined as product of three factors: availability, performance rate and quality rate.

\subsection{Causal Chain to OEE}

OEE can be linked to major equipment losses. Major equipment losses can be categorized as six big losses. The linkages between OEE factors [3, 4] and the six big losses are illustrated in Fig. 1. Time losses can be divided into downtime, speed and quality losses. [5]. 


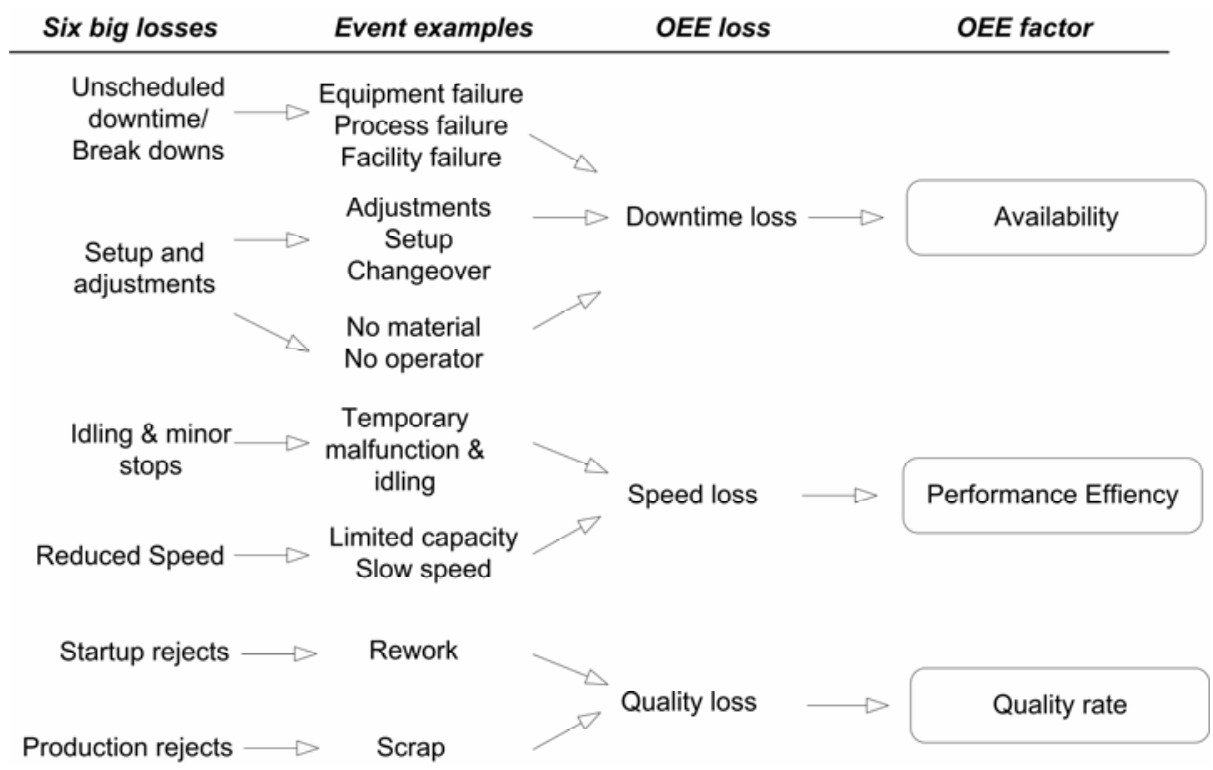

Fig. 1. Linkage between OEE factors and six big losses. (adapted from [3, 4]).

\subsection{OEE Extended to Operations Performance Assessment}

OEE is not an explicit index of performance of a manufacturing process. OEE can be seen as an indicator how well equipment or machinery are performing or are been utilized at a specified time frame and indicate, which parts of assembly line or machinery is not performing well. As stated in [6]: "the most important objective of OEE is not to get an optimum measure, but to get a simple measure that tells the production personnel where to spend their improvement resources".

OEE can be used to fully automated and semi-automated manufacturing lines. In automotive industry type of manufacturing lines vary from fully automatic to manually operated stations, thus there are possibilities to utilize OEE as a tool. In OEE, the machinery is assumed to generate a fixed ideal cycle time and due to changing resources in manual assembly, accurate measurement is challenging.

The purchase price of equipment is just one cost element in the comparison [7]. Total Cost of Ownership (TCO) methodology has shown how important it is to analyse all the cost, direct and indirect, incurred throughout the life cycle of an equipment, including acquisition and installation, operations and maintenance, and end-of-life management. TCO methodology pinpoints costs that could be easily underestimated, such as quality and rework as well as all the costs of running the system. The methodology is useful in system integrator and end-user collaboration, where both can use similar formulae in system evaluation and trade-off analysis. 


\section{Methodology and Data}

In the rear screen installation case main benefits of applying COBOT are: improved productivity, improved quality, reduced production cost and improved ergonomics.

High flexibility and improvements in ergonomics are the two major reasons for applying assistant robots. Better ergonomics is expected to improve production quality and production rate [8]. Production quality and production rate are possible to be measured in terms of OEE.

\subsection{Definition of OEE Parameters}

Usefulness of OEE in relies on reliability of measurements or numbers from which OEE factors are calculated. Some variables are usually available from factory databases. However this data is not always accurate, thus values of OEE factors are not necessarily accurate enough e.g. for benchmarking purposes. This especially concerns calculation of availability variables; accurate down times are typically unknown. If accurate OEE is required, calculation of the variable values should be based on accurate numbers or measurements collected manually or automatically from the assembly line.

There may exist also dependencies between the OEE factors or variables. Increasing performance efficiency may decrease quality and lower availability may also be seen as lower performance efficiency. Raising efficiency of one factor in cost of another is not always acceptable. For instance, increase in performance efficiency in cost of quality is typically unacceptable.

An improvement of one variable may also be insufficient. For instance, decreasing only planned cycle time would decrease performance efficiency, if number of manufactured products were not increased. If this is not possible, availability efficiency should be increased by reducing set-up times and down times. In flexible assembly lines where product volumes or type may vary it is important to affect to several variables to maintain good performance level of the line. A practical way to increase the performance of the line is to focus continuous improvements in one variable at a time (availability parameters) i.e. improvements are focused to reduce first setup times, then down time etc.

Especially important in this context is to have tools for human-COBOT cooperation life cycle monitoring. The concept should enable the validation of benefits of new COBOT solutions and give clear indications for continuous development processes. Special emphasis should be put on generating, delivering and using information about human-COBOT cooperation performance. Human creativity, intelligence, knowledge, flexibility, and skills are hardly directly transformed to performance indices. Instead, indirect asset utilisation and operational efficiency indicators must be found.

Since human and COBOT are working co-operatively human actions have also affects on process performance, i.e. OEE. However a human's and machines or a COBOT's efficiency are undistinguishable directly from OEE factors. It is possible to infer cause of lower efficiency from the variables from which OEE factors are calculated. One such variable is cycle time, which is used to define performance efficiency. In addition, availability of operators affects calculation of availability. 


\subsection{Measuring of OEE Parameters}

The automatic measurement of accurate availability parameters such failure times and limited availability of operators and material can be difficult. For instance, there is no way to directly measure availability of an operator. Many cases down times are thereby collected manually in order to calculate accurate values. In manual collection operators write down times and their reasons into paper forms or records them into a computer program. This is carried out along with their normal activities. Manually collected down times can also be inaccurate [9]. Operators can be unmotivated for recording these times, there is no time to record them or the operators forget to record them as well as measuring of the down times can be difficult.

Automatic data collection of down times would be much more attractive. Down times could be defined indirectly from machine measurements or events. Sensors can measure conveyor speeds, electric motors on/off times, passing time of a part or a component passing through an assembly station etc. Many of these variables are already available from factory automation systems or data bases. However, installation of new sensors is probably needed. The sensors for measuring above mentioned information are usually inexpensive. Cost of installation of sensors and their hard wiring could be minimized by using wireless data transfer and feeding power from batteries. Also moving of wireless sensors nodes to other locations is easy. Fig. 2 represents OEE data collection, where production data management gives information about defects in manufacturing. Production line is not usually stopped when defects occur, and so those events are stored into a database on a later phase. Therefore that information is not available real time. Automation systems give information about time-based productivity measurements. Unscheduled downtimes are manually entered to the information system at the COBOT cell.

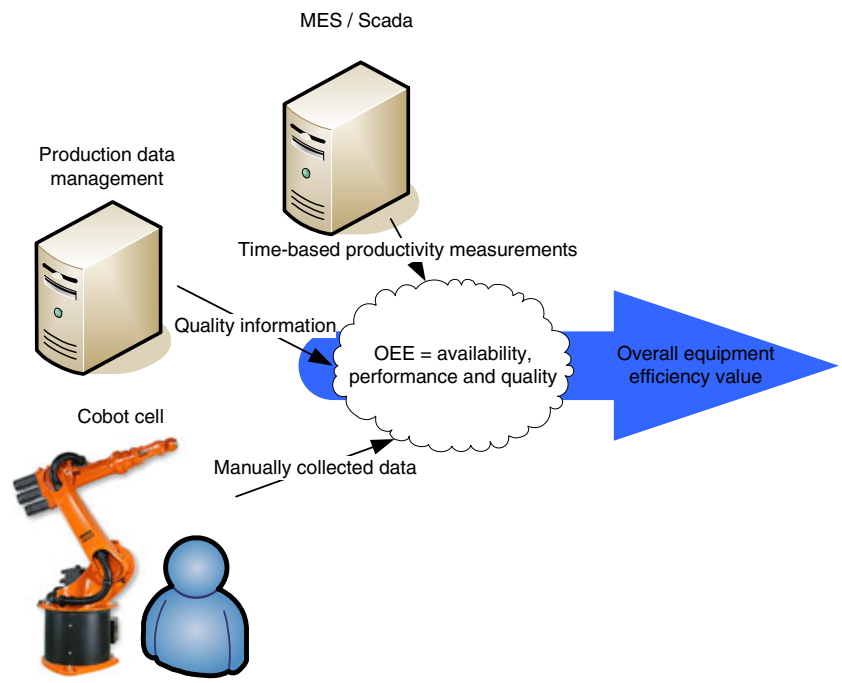

Fig. 2. Collecting OEE variables from different sources 
What to measure in order to define cycle times? The rear screen installation case is used here as an example. Cycle time of rear screen installation could be defined as follows:

The window installation process begins when the COBOT grabs a window from a panel supply and begins to move towards a vehicle with its suction pads attached to a window panel. COBOT trajectories and its events are possible to be stored into a database. Cycle time can be measured from the database by using the preceding terms.

What to measure in order to define down times? Down times have been separated into two categories in the six big losses -definition. Breakdowns consist of tooling failures, unplanned maintenance events and general breakdowns. Down times regarding setup and adjustment consist of setup and changeover tasks, material shortages, operator shortages, major adjustments of the equipment and warmup-time of the equipment.

A way to define operating times is to subtract a machine's down time from loading times [3]. Loading time is the time when the machine is in productive use. In practice measuring of several machines or their components states may be needed in order to calculate operating times. An inferences mechanism for defining states of the machines may also be needed.

Down times can be measured by using the COBOT trajectory database. Some reasons for idle times and down times may be derived also from the COBOT trajectory database. For example a person entering the COBOT's safe area will halt the COBOT. The reason for halting is stored into the database.

\section{Findings}

By introducing of COBOT to an assembly task, it is expected to increase quality rate and performance efficiency. Quality in terms of OEE factors can be monitored by measuring variables from which OEE factors are calculated. However OEE is not used for controlling robot or task execution i.e. there is no feedback to the systems. It is just a measure how well a machinery or process is performing. Since human and COBOT are working co-operatively human actions have also affects on process performance, i.e. OEE. However a human's and machines or a COBOT's efficiency are undistinguishable directly from OEE factors. It is possible to infer cause of lower efficiency from the variables from which OEE factors are calculated. One such variable is cycle time, which is used to define performance efficiency. From the monitoring point of view this means, that a monitor catching the events denoting the start and end of the assembly sequence, showing active time of the assembly sequence and idle time between the assemblies is used.

\section{Conclusions}

OEE is one life-cycle parameter and it offers a tool to evaluate the effects of introducing new machinery in the performance of a manufacturing line. Especially OEE offers a tool to evaluate effects of continuous improvements i.e. where to focus maintenance 
and improvements activities. The actual suitability of OEE in selected case (car rear screen assembly) should be tested in the real work environment with an OEE tool.

Acknowledgements. This work is partially funded by the European Union as part of the NMP-2004-3.4.3.12 PISA project.

\section{References}

1. Hägele, M., Schaaf, W., Helms, E.: Robot Assistants at Manual Workplaces: Effective Cooperation and Safety Aspects. In: Proceeding of the 33rd International Symposium on Robotics 2002, Stockholm, Sweden (2002)

2. Bernhardt, R., Surdilovic, D., Katschinski, V., Schreck, G., Schröer, K.: Next Generation of Flexible Assembly Systems. In: Innovation in Manufacturing Networks IFIP International Federation for Information Processing, vol. 266, pp. 279-288. Springer, Boston (2008)

3. Dal, B., Tugwell, P., Greatbanks, R.: Overall equipment effectiveness as a measure of operational improvement - A practical analysis. International Journal of Operations \& Production Management 20, 1488-1502 (2000)

4. Pomorski, T.: Managing Overall Equipment Effectiveness (OEE) to Optimize Factory Performance. In: 1997 IEEE International Symposium on Semiconductor Manufacturing Conference Proceedings, pp. A33-A36. IEEE, New York (1997)

5. Högfeldt, D.: Plant Efficiency. A value stream mapping and overall equipment effectiveness study, Master's thesis, Luleå University of Technology (2005)

6. Tangen, S.: An overview of frequently used performance measures. Work Study 52, 347 354 (2003)

7. Heilala, J., Montonen, J., Helin, K.: Selecting the right system - assembly system comparison with total cost of ownership methodology. Assembly Automation 27, 44-54 (2007)

8. Akella, P., Peshkin, M., Colgate, E., Wannasuphoprasit, W., Nagesh, N., Wells, J., Holland, S., Pearson, T., Peacock, B.: Cobots for the automobile assembly line. In: Proceedings of the 1999 IEEE International Conference on Robotics \& Automation, pp. 728-733. IEEE, New York (1999)

9. Ljungberg, O.: Measurement of overall equipment effectiveness as a basis for TPM activities. International Journal of Operations \& Production Management 18, 495-507 (1998) 